\title{
A comparative analysis of two unrelated outbreaks in Latvia and Australia of acquired idiopathic megaesophagus in dogs fed two brands of commercial dry dog foods: 398 cases (2014-2018)
}

\author{
Karyl J. Hurley DVM \\ Caroline Mansfield BSc, BVMS, PhD \\ Ilze Matīse VanHoutan DVM, PhD \\ Lauren Lacorcia BVSc, MVSc \\ Karin Allenspach Dr Med Vet, PhD \\ Geoffrey Hebbard B Med Sci, MBBS, PhD \\ Stanley L. Marks BVSc, PhD \\ Robert Poppenga DVM, PhD \\ James H. Kaufman $\mathrm{PhD}$ \\ Bart C. Weimer PhD \\ Kevin D. Woolard DVM, $\mathrm{PhD}$ \\ Joe Bielitzki DVM, MS \\ Derek Lulham BSc \\ Jerome Naar PhD
}

From Scientific and Regulatory Affairs, Mars Inc, McLean, VA 22I0I (Hurley); Faculty of Veterinary and Agricultural Sciences, Melbourne Veterinary School, The University of Melbourne, Werribee, VIC 3030, Australia (Mansfield, Lacorcia); Riga, Latvia (Matīse VanHoutan); Department of Veterinary Clinical Sciences, College of Veterinary Medicine, lowa State University, Ames, IA 500II (Allenspach); Department of Gastroenterology and Hepatology, The Royal Melbourne Hospital, Melbourne, VIC 3050, Australia (Hebbard); Department of Medicine and Epidemiology (Marks), California Animal Health and Food Safety Laboratory (Poppenga), Department of Population Health and Reproduction (Weimer), and Department of Pathology, Microbiology, and Immunology (Woolard), School of Veterinary Medicine, University of California-Davis, Davis, CA 95616; IBM Almaden Research Center, San Jose, CA 95120 (Kaufman); National Aeronautics and Space Administration, Merritt Island, FL 32953 (Bielitzki); Sourcing Quality Management, Mars Inc, Sydney, NSW 2060, Australia (Lulham); and Mars Petcare, 30470 Aimargues, France (Naar).

Address correspondence to Dr. Hurley (karyl.hurley@ effem.com).

\begin{abstract}
CASE DESCRIPTION
In Latvia in 2014, acquired idiopathic megaesophagus (AIME) was observed in increased numbers of dogs that consumed varieties of $I$ brand of dog food. Within 2 years, 253 dogs were affected. In Australia in November 2017, 6 working dogs that consumed I diet of another brand of dog food developed AIME. In total, 145 Australian dogs were affected.
\end{abstract}

\section{CLINICAL FINDINGS}

AIME was diagnosed predominantly in large-breed male dogs (> $25 \mathrm{~kg}[55$ lb]). Regurgitation, weight loss, and occasionally signs consistent with aspiration pneumonia (coughing, dyspnea, or fever) were noted. Most Latvian dogs had mild to severe peripheral polyneuropathies as evidenced by laryngeal paralysis, dysphonia, weakness, and histopathologic findings consistent with distal axonopathy. In Australian dogs, peripheral polyneuropathies were not identified, and histopathologic findings suggested that the innervation of the esophagus and pharynx was disrupted locally, although limited samples were available.

\section{TREATMENT AND OUTCOME}

Investigations in both countries included clinical, epidemiological, neuropathologic, and case-control studies. Strong associations between the dog foods and the presence of AIME were confirmed; however, toxicological analyses did not identify a root cause. In Latvia, the implicated dietary ingredients and formulations were unknown, whereas in Australia, extensive investigations were conducted into the food, its ingredients, the supply chain, and the manufacturing facilities, but a cause was not identified.

\section{CLINICAL RELEVANCE}

A panel of international multidisciplinary experts concluded that the cause of AIME in both outbreaks was likely multifactorial, with the possibility of individualized sensitivities. Without a sentinel group, the outbreak in Australia may not have been recognized for months to years, as happened in Latvia. A better surveillance system for early identification of pet illnesses, including those associated with pet foods, is needed. ( $\mathrm{Am}$ Vet Med Assoc 2021;259:172-183)

\section{Latvian Outbreak 2014 to 2016}

In the first quarter of 2015, veterinarians in Latvia observed a year-over-year increase in dogs that had

$\begin{array}{ll}\text { ABBREVIATIONS } \\ \text { AAFCO } & \text { Association of American Feed Control Officials } \\ \text { AChE } & \text { Acetylcholinesterase } \\ \text { AIME } & \text { Acquired idiopathic megaesophagus } \\ \text { BEA } & \text { Beauvericin } \\ \text { EMG } & \text { Electromyography } \\ \text { ME } & \text { Megaesophagus } \\ \text { MNCV } & \text { Motor nerve conduction velocity } \\ \text { MPA } & \text { Mars Petcare Australia } \\ \text { PetFAST } & \text { Pet Food Adverse Event System of Tracking } \\ \text { PNP } & \text { Peripheral polyneuropathy } \\ \text { UoM } & \text { University of Melbourne }\end{array}$

been diagnosed with AIME. Prior to 2014, the expected annual incidence was approximately 10 to 15 cases/y. Prompted by the observed increase in dogs with AIME, one of the authors, a board-certified veterinary pathologist (IMVH), led an investigation of this situation by means of 1 retrospective and 2 prospective studies that included a prospective case-control study. ${ }^{1, a}$ The case-control study was approved by the Latvian Minister of Agriculture. Funding for the studies was initially provided through a grant from the Minister of Agriculture and later through crowdsourcing. Performed in May 2015, the retrospective study was an epidemiological investigation of possible causes of AIME. Findings indicated no correlations among geographic region, presence of infec- 
tion, vaccination status, or administration of prophylactic parasiticides. However, 94\% of affected dogs had been consuming the same brand of locally produced and distributed dry dog food, Brand X. ${ }^{\mathrm{b}}$ Therefore, diet was evaluated in the prospective studies. Brand $\mathrm{X}$ was labeled for adult dogs, and puppies were not reported to have been affected. All 7 varieties (including for small dogs and senior dogs and basic and pro lines), regardless of protein source (chicken, lamb, or meat), of Brand $\mathrm{X}$ appeared to be associated with AIME. The varieties of Brand $\mathrm{X}$ varied in their composition, and Brand $\mathrm{X}$ was generally considered an economy food (ie, considerably lower priced than other dog food brands). According to Brand X's label of the variety with meat, Brand $\mathrm{X}$ was composed of $50 \%$ cereals, $20 \%$ processed animal proteins, 6\% oil and fat, and $2.1 \%$ vitamin-microelement supplement, which equated to $25.5 \%$ protein, $10 \%$ fat, $7 \%$ ash, $3.5 \%$ fiber, $1.5 \%$ calcium, and $1.3 \%$ phosphorus on a drymatter basis.

Owner consent was obtained for inclusion of their dog in the studies and for the use of collected data for the sole purpose of the studies. All studies included electronic distribution of questionnaires to practicing veterinarians to collect the results of a case dog's physical examination and dog owner-provided history, including the dog's home environment and feeding practices; administration of any flea, tick, and endoparasite preventives; and vaccinations. Questionnaires were also made available through the Latvian Association of Veterinarians' website. Questionnaires were completed and submitted electronically through a platform ${ }^{\mathrm{c}}$ that provided a time stamp and saved the original record. When needed, follow-up by phone was undertaken. For the 3 studies, a case was defined as a dog that had confirmed ME by means of thoracic radiography, necropsy, or both. For the prospective case-control study, a control was defined as a dog that was $\geq 1$ year of age and did not have clinical signs consistent with ME. The age of $\geq 1$ year was required for study enrollment of control dogs because the retrospective study had shown that dogs $\geq 1$ year old were affected in the outbreak. This also ensured that dogs with congenital ME were not inadvertently included. A veterinarian who specialized in diagnostic imaging reviewed all radiographs to confirm the presence of ME. If a second opinion was needed to confirm the presence of ME, radiographs were sent to a board-certified radiologist for review. Some dogs that were suspected to have had ME on the basis of clinical signs were excluded because radiographs were of poor quality to confirm ME or because ME that had been diagnosed at the primary care clinic was not confirmed by the specialists.

Between 2014 and 2016, 253 dogs (mean age, 6.4 years; range, 1 to 12 years) met the definition of a case. Most affected dogs were male (192/253 [76\%]) and mixed breed (76/253 [30\%]). Questionnaire response rate of the owners of case dogs for the case-control study was $91 \%(64 / 70)$. Case dogs were of various breeds, including $\geq 3$ dogs/breed for each of the following breeds: Bernese Mountain Dog, Central Asian Shepherd dog, Dachshund, Doberman Pinscher, German Shepherd Dog, Labrador Retriever, and Rottweiler. Control dogs included $\geq 3$ dogs/breed for each of the following breeds: Bernese Mountain Dog, Central Asian Shepherd Dog, Dachshund, German Shepherd Dog, and Yorkshire Terrier. Regurgitation, dyspnea, dysphonia, and weakness were the most common clinical signs, and all dogs had radiographic evidence of generalized ME, such that the term ME-PNP was used to describe this outbreak. On the basis of detailed analyses of blood and tissue samples, possible causes of ME-PNP that were ruled out were canine distemper, dysautonomia, hypothyroidism, myasthenia gravis, neosporosis, and various toxicoses (acrylamide, botulism, heavy metal, ionophore, pesticides [those most commonly used], and thallium).

Esophageal tissue and other organ tissues, if they had not greatly autolyzed, were collected for microscopic examination from each dog that had died or had been euthanized and that had undergone a complete necropsy. As the study progressed, a polyneuropathy was apparent for the dogs that had ME; therefore, numerous neuromuscular tissues were specifically sampled during the necropsy. For a subset of case dogs $(n=19)$, the collected neuromuscular tissues were as follows: brachial plexus, phrenic nerve, the cervical and distal portions and dorsal and ventral branches of the vagus nerve, facial nerve, lingual nerve, hypoglossal nerve, recurrent laryngeal nerve, cranial laryngeal nerve, peroneal nerve, ulnar nerve, sciatic nerve, tongue, temporal muscles, masticatory muscles, laryngeal cricothyroid muscle, diaphragm, triceps brachii muscle, gluteus medius muscle, biceps femoris muscle, cervicothoracic ganglion, distal ganglion of the vagus nerve, paravertebral ganglion between the second and third ribs, forebrain (cerebrum, thalamus, and hypothalamus), midbrain, hind brain (medulla oblongata, pons, and cerebellum), and cervical portion of the spinal cord. Histologic evaluation of the peripheral nerves indicated a distally enhanced intermediate axonopathy with subtle to massive changes in axons and myelin sheaths and clear proximodistal advancement. The most severely affected nerves were those that were the longest, which were the vagus nerve and its branches (eg, recurrent laryngeal nerve) and the sciatic nerve. Nerve lesions widely varied in severity, most commonly affecting small-diameter motor nerves of the distal portion of the limbs (vs proximal portion) and the pelvic limbs (vs thoracic limbs). Variable extensive skeletal muscle fiber atrophy, including the striated muscle of the esophagus, was noted secondary to the nerve lesions.

In July 2016, a board-certified veterinary neurologist examined a subset $(n=11)$ of case dogs. The most frequent findings were decreased spinal reflexes for all limbs $(n=8)$ and generalized muscle atrophy (7). Electromyography was performed for $10 \mathrm{dogs}$ and 
used to assess 11 limb muscle groups on the right side of the body. One dog was not evaluated with EMG because of its frequent bouts of aspiration pneumonia and the dog owner's concern about aspiration of esophageal contents and development of pneumonia during or after general anesthesia required to perform EMG. The 10 dogs had abnormalities in the muscles of the pelvic limbs, and similar abnormalities were detected in the muscles of the thoracic limbs in 7 dogs. The most common abnormalities were fibrillation potentials and positive sharp waves and, less commonly, prolonged insertion potentials. Nine dogs had reduced MNCV of the sciatic nerve between 2 stimulation sites.

In general, electrophysiologic and clinical findings indicated that all case dogs had various degrees of peripheral nerve damage consistent with distal polyneuropathy, characterized by more severe, consistent lesions in the nerves and muscles distal to (vs proximal to) the stifle and elbow regions. Likewise, histologic lesions of the nerves correlated well with neurologic, EMG, and MNCV findings in terms of lesion distribution and damage type. Esophageal lesions were predominantly muscle fiber degeneration and atrophy. Mononuclear esophagitis was present in 4 of 30 (13\%) dogs, with mucosal erosions in $1 \mathrm{dog}$. Loss of cells of the esophageal ganglion was equivocal. Clinically relevant lesions in the brain, cervical portion of the spinal cord, and spinal ganglia were not noted.

The brain tissue samples from 11 dogs were submitted to a veterinary diagnostic laboratory for determination of AChE activity to rule out organophosphate and carbamate toxicity (these insecticides inhibit AChE). ${ }^{\mathrm{d}} \mathrm{A}$ marked reduction in AChE activity was detected in the samples of 9 of these dogs (Michel method; range, 0.04 to 0.18 delta $\mathrm{pH}$ units/h; values $<0.21$ delta $\mathrm{pH}$ units $/ \mathrm{h}$ considered positive [ie, AChE activity inhibited], and value of 0.43 delta $\mathrm{pH}$ units/h considered normal). Furthermore, in case dogs, AChE inhibition was not detected in the blood, and organophosphate and carbamate insecticides were not detected in the serum, liver, and brain by gas chromatography-mass spectroscopy. Decreased AChE activity may have been because of tissue autolysis, but autolysis was not evident; yet biological samples were analyzed after storage at $-20^{\circ} \mathrm{C}\left(-4^{\circ} \mathrm{F}\right)$, and reduced activity because of storage could not be excluded.

For the case-control study with 64 case dogs and 123 randomly selected control dogs (dogs without ME between April and June 2016 and according to inclusion and exclusion criteria, selected by simple random procedure), the odds, determined with multivariable logistic regression analysis, of case dogs that were fed only Brand X developing ME-PNP were markedly increased (OR, 356; 95\% CI, 63 to 2,020). Dogs fed Brand $\mathrm{X}$ dog food in addition to other foods were 81 (95\% CI, 14 to 468) times as likely to be a case, and this fact indicated a possible dose effect in the development of ME-PNP.
Multivariable logistic regression analysis included body weight as a confounding factor. Analysis included the number of brands of foods fed, the consistency of the foods (dry vs wet [canned]), and whether supplements were provided. Case dogs were more commonly fed 1 brand of dog food (89\%), compared with control dogs (59\%). Also, $44 \%$ of case dogs were fed only commercial dry dog food, compared with $15 \%$ of control dogs. For both case and control dogs, the proportion consuming wet food was approximately $10 \%$. Feeding a homemade diet irrespective of the proportion of the entire diet that was homemade (homemade food $<10 \%$ or $\geq 10 \%$ of the entire diet) was more frequent for control dogs $(72 \%)$ versus case dogs (48\%). Control dogs were more often fed raw meat than case dogs ( $37 \%$ vs $14 \%$, respectively). Vitamins and feed supplements were provided less often to case dogs, compared with control dogs (11\% vs $36 \%$, respectively).

Ten samples of Brand $\mathrm{X}$ that represented 10 lots and blood samples from 10 case dogs were analyzed for concentrations of vitamins B1, B6, and B12. ${ }^{\mathrm{e}}$ Vitamin concentrations in Brand $\mathrm{X}$ were within the range the AAFCO recommended, but their concentrations were a sixth to a tenth those of another commercial

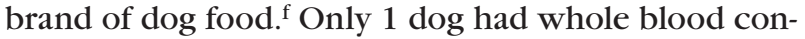
centrations of vitamins $\mathrm{B} 1$ and $\mathrm{B} 6$ and serum concentration of vitamin B12 within reference limits. Of the remaining 9 dogs, 2 had low whole blood concentrations of vitamin B1 (range, 12 to $20.5 \mu \mathrm{g} / \mathrm{L}$; reference interval, 46 to $112 \mu \mathrm{g} / \mathrm{L}), 7$ had low whole blood concentrations of vitamin B6 (range, 1.2 to $9.9 \mu \mathrm{g} / \mathrm{L}$; reference interval, $>13 \mu \mathrm{g} / \mathrm{L}$ ), and 7 had low serum concentrations of vitamin B12 (range, $<50$ to $262 \mathrm{pg}$ / $\mathrm{mL}$; reference interval, 300 to $800 \mathrm{pg} / \mathrm{mL}$ ). ${ }^{\mathrm{g}}$

With a diet change and feeding from an elevated platform, some case dogs partially improved but continued to suffer from generalized weakness and bouts of aspiration pneumonia. Megaesophagus remained in the dogs that had follow-up radiography; however, complete recovery was seen in $1 \mathrm{dog}$, which may have indicated that ME was diagnosed earlier than for other dogs.

The epidemic curve (ie, a visual representation often in the form of a graph that depicts the onset and progression of a disease outbreak) together with analysis of the lag time between the onset of clinical signs and the diagnosis of ME-PNP (2 to 3 months) suggested that the mean time from initial exposure to onset of clinical signs was approximately 6 to 12 months and that the outbreak should cease 6 to 12 months after market withdrawal or a marked reduction in sales of Brand $\mathrm{X}$.

In the summer of 2016 as veterinarians and social media posts warned the general public of the MEPNP outbreak, case numbers decreased in parallel with reduced sales of Brand X (decreased from 200 tons/mo to 50 tons $/ \mathrm{mo}$ ). ${ }^{\mathrm{h}}$ Presumably Brand $\mathrm{X}$ was reformulated because its odor and appearance changed for the lots produced after 2016; also, the lot numbers started from 1 at the beginning of 2016. 
In all, 253 dogs of an estimated 33,000 dogs that reportedly consumed Brand $\mathrm{X}$ in Latvia met the case definition (for years 2014 to 2016). ${ }^{a}$ Mortality rate for case dogs was $20 \%$ to $25 \%$ because of aspiration pneumonia, a marked decrease in body weight, muscle atrophy, or progressive weakness. Much of the Latvian countryside was remote, and radiographic equipment was not available in every veterinary clinic; therefore, some owners of case dogs may not have had access to veterinary care or their dogs may have been diagnosed on the basis of clinical signs alone such that they were not or could not be registered in the 3 studies because 1 inclusion criterion was radiographic confirmation of ME. At the annual Latvian small animal practitioner meeting in 2016, approximately $90 \%$ of veterinarians in attendance raised their hand when one of the authors (IMVH) asked whether they had diagnosed ME-PNP in dogs in their practices. Because of the aforementioned reasons, the number of affected dogs in the outbreak was likely underestimated. The data regarding the strong link between the ME-PNP outbreak and consumption of Brand $\mathrm{X}$ were presented to its manufacturer, but the manufacturer denied the link and access to information on recipes and ingredients.

In addition to analyzing Brand $\mathrm{X}$ for vitamins $\mathrm{B} 1$, $\mathrm{B} 6$, and $\mathrm{B} 12$, Brand $\mathrm{X}$ was also analyzed for mycotoxins, urea, and other toxicants, including acrylamide, heavy metals (iron, manganese, and thallium), ionophores, magnesium, and pesticides. Low concentrations of fumonisins (0.1 to $0.5 \mathrm{ppm}$ ) and vomitoxin (0.2 to $0.5 \mathrm{ppm})$ were detected as well as low concentrations of urea in some samples. Concentrations of iron, magnesium, and manganese were comparatively high ( $2 \mathrm{X}$ to $6 \mathrm{X}$ the concentrations recommended by the AAFCO) but were not high enough to cause concern about toxicosis. An ELISA and a PCR assay were not available for food analysis. However, testing for pathogenic bacteria with standard culture methods yielded negative results, and testing on cell cultures did not indicate a toxic effect on cells, but inhibition of cell growth was noted with some of the samples. A limited number of food samples was saved for future analysis. To date, however, an international team of multidisciplinary veterinary experts who have been consulted has not yet identified a reason for the association between consumption of Brand $\mathrm{X}$ and the development of ME-PNP.

\section{Australian Outbreak 2017 to 2018}

In late October and early November 2017, 6 male working dogs from the same work unit in Victoria, Australia, developed ME over an 8-week period. The initial clinical sign was regurgitation and, for $1 \mathrm{dog}$, fever, likely associated with aspiration pneumonia. These dogs were part of a facility that provided specialized training to 60 working dogs. Five of the dogs that developed ME were German Shepherd Dogs, 2 of which were littermates, and 1 was a Labrador Retriever. They resided with their handlers yet had access to common training and working facilities approximately $10 \%$ of the time. They received the same flea, tick, and heartworm preventives and were fed various brands of dry dog food. When considering all dogs at the facility, 18 were fed Diet Y dry dog food, ${ }^{\mathrm{i}}$ and 6 of 18 were affected with ME. No concerning environmental or physical factors were identified. The working dog facility commissioned one of the authors (CM) at the UoM to investigate this cluster of ME in January 2018. ${ }^{2}$ Investigations failed to identify any infectious, endocrine, or autoimmune cause, such that Diet $\mathrm{Y}$ was considered a possible cause, and feeding of it was immediately discontinued.

Mars Petcare Australia, the manufacturer of Diet Y, which was one of several diets under the same brand name, was contacted by personnel at the working dog facility in late December 2017 to notify them of their concerns; MPA's Quality and Food Safety team initiated a detailed investigation of the formulation, raw materials (ingredients), and manufacturing conditions of the identified production runs of Diet $\mathrm{Y}$ that was being fed to the affected dogs. Diet Y was produced in Bathurst, NSW, Australia, and distributed throughout Australia. All test results and manufacturing conditions were determined to be normal. In midFebruary 2018, 2 working Labrador Retrievers from South Australia were affected with ME, and samples from the lots of Diet Y fed to these dogs were sent to laboratories ${ }^{\mathrm{j}, \mathrm{k}}$ for toxicological testing, including for acrylamide, heavy metals, and pesticides. No toxicants of concern were identified. In early March 2018, MPA and UoM conferred with PetFAST (a voluntary joint initiative of the Australian Veterinary Association and the Pet Food Industry Association of Australia for the monitoring of health problems in dogs and cats suspected of being associated with pet foods) about this cluster of cases, and at that time no other such cases had been reported.

Subsequently, the UoM issued a statement to veterinarians through the Australian Veterinary Association that requested they report cases to the UoM, and although no new cases were immediately reported after issuance of the statement, media coverage raised public awareness of the situation. With the addition of UoM, veterinarians and dog owners reported cases through 3 entities: UoM, MPA, and PetFAST. All case information reported through MPA and PetFAST was sent to the UoM for collation and analysis. On March 23,2018 , UoM was contacted by a veterinarian regarding a cluster of pet dogs with ME that were fed Diet $\mathrm{Y}$, including 2 dogs from the same household. This cluster of cases was immediately reported to MPA, and within 24 hours of the report, MPA voluntarily recalled Diet $\mathrm{Y}$ and manufacturing and shipping of Diet Y ceased. Consumers across the country were alerted to the recall through statements on the MPA website and social media channels, from retailers and veterinarians, and on traditional media. Geographic distribution of affected dogs was widespread across Australia. Tissue samples were not harvested from af- 
fected dogs, or harvested samples were not adequately preserved for analysis.

The 8 affected working dogs and 3 affected pet dogs were evaluated at the UoM. The diagnostic tests for all dogs were as follows: physical and neurologic examinations, thoracic radiography, barium swallow video fluoroscopy, and analyses of a serum sample to rule out causes of ME such as hypoadrenocorticism (serum basal cortisol concentration or serum cortisol concentration before and after ACTH stimulation), hypothyroidism (serum total and free thyroxine hormone concentration with or without thyroid-stimulating hormone concentration), and myasthenia gravis (acetylcholine receptor antibody titer). Electromyography and pharyngoesophagoscopy were performed for one dog and pharyngoesophagoscopy for another dog. One of the 2 dogs had mild laryngeal paralysis determined by inspection of the arytenoid cartilages while the dog was lightly anesthetized, but this dog did not have a change in vocalization or have stridor; therefore, this finding was not considered clinically important. Histologic evaluation of esophageal biopsy specimens collected from the dog that underwent only pharyngoesophagoscopy revealed the presence of mucosal ulceration and neutrophilic inflammation. All dogs had regurgitation, with the primary physical examination finding of poor body condition and, for some dogs, clinical signs consistent with aspiration pneumonia. Neurologic examination was unremarkable for each dog. One dog had radiographic evidence of aspiration pneumonia. Dogs did not have evidence of concurrent disease on the basis of the results of the aforementioned diagnostic tests, such that these dogs were subsequently diagnosed with AIME.

Four dogs had postmortem examinations performed at the UoM, and none shared a common lesion that could be the cause of ME. No dog had identifiable lesions in the brain, spinal cord, or peripheral nerves that innervate the limbs. The postmortem examination of 1 dog that was performed during the early period of the outbreak revealed multifocal neurogenic atrophy of the esophageal and laryngeal muscles, unremarkable recurrent laryngeal nerves, and mild gastritis and moderate enteritis (mixed inflammatory infiltrates). The postmortem examination of $1 \mathrm{dog}$ that was performed shortly after the recall of Diet Y revealed severe skeletal muscle atrophy of the distal portion of the esophagus. The local nerve fibers in the esophageal tissue appeared normal, but distal axonopathy in the recurrent laryngeal nerves and axonal degeneration of the terminal nerve endings in the gastric cardia were evident. The left and right cricoarytenoid muscles had multifocal neurogenic atrophy. The third and fourth dogs were euthanized $>12$ months after the recall. The third dog had generalized nonneurogenic, noninflammatory myofiber degeneration and necrosis throughout the esophagus and unremarkable laryngeal muscles. The fourth dog had mild generalized myofiber degeneration in the esophagus, possibly secondary to overdistention, with minimal evidence of a laryngeal neuropathy.
The variable pathological changes may have indicated that the development of AIME was multifactorial and the duration of AIME was variable among the dogs.

Although the esophageal mucosa was grossly normal in appearance in the first 2 dogs necropsied at the UoM, histologic examination revealed subacute mucosal ulceration and neutrophilic infiltration and multifocal mild to moderate esophagitis. In addition to the previously mentioned 4 dogs that underwent necropsy, rare microscopic erosions and neutrophilic infiltration were present in the esophagus of a working dog that was necropsied at the University of Adelaide. Taken in conjunction with the endoscopic biopsies, 4 of 6 of the dogs evaluated by endoscopy had mucosal erosion and ulceration of the distal portion of the esophagus. This was thought to be secondary to mucosal irritation caused by the retention of food, regurgitation, or the kibble structure. No dog was reported to have had signs of PNP, laryngeal paralysis, or dysphonia. One dog that had been evaluated at the UoM had resolution of ME as determined by barium swallow video fluoroscopy; this dog may not have been as severely affected as the other dogs.

As with the Latvian outbreak, the UoM conducted a matched case-control study to identify risk factors for the development of AIME. ${ }^{3}$ Also, the VetCompass Australia database was accessed to determine the incidence of AIME in an attempt to verify the supposition that AIME was associated with consumption of Diet Y. VetCompass Australia is a database of de-identified demographic and medical information from pet owner consultations and pet examinations from hundreds of veterinary clinics around Australia. ${ }^{4}$ Veterinary researchers can mine the database to obtain a baseline of expected incidence and unexpected increased incidence, indicative of an outbreak, of various diseases. After institutional human ethics approval, questionnaires were designed to collect data on the dogs that were reported to the UoM with clinical signs consistent with ME and confirmed radiographically with AIME by one of the authors (CM) and each dog's attending veterinarian between July 1, 2017, and April 30, 2018. Questionnaire details for 77 cases and 121 controls (ratio, approx 1:1.6) were available for analysis via a secure web application for building and managing online surveys and databases. ${ }^{5,6}$ Controls were selected by choosing 3 dogs that were presented to the referring practice on the same day. For the cases, the median date of onset of clinical signs (date when $50 \%$ of the cases were enrolled in this case-control study) was January 27, 2018. Fortynine $(64 \%)$ case dogs were male (neutered, $\mathrm{n}=43$; sexually intact, 6), and 30 (38\%) case dogs were female (neutered, 26; sexually intact, 2). Case dogs had a median age of 6.6 years (interquartile [25th to 75 th percentile] range, 4.6 to 8.7 years) and a median body weight of $30.2 \mathrm{~kg}$ ( $66.4 \mathrm{lb}$; interquartile range, 24.6 to $39.3 \mathrm{~kg}$ [54.1 to $86.5 \mathrm{lb}$ ]). Breeds that were overrepresented as case dogs were Dalmatian, German Shepherd Dog, Golden Retriever, and Labrador Retriever, all large-breed dogs commonly affected by 
allergic skin disease and AIME.,8 Correlations were not significant between the presence of AIME and geographic region; administration of flea, tick, and endoparasite preventives; brand of vaccine with antigens of canine distemper virus, canine adenovirus, canine parainfluenza virus, and canine parvovirus; or timing of vaccine administration.

This case-control study showed that dogs with AIME were 437 (95\% CI, 80 to 2,361) times as likely to have had a recent history of consuming Diet Y, compared with control dogs. ${ }^{3}$ Furthermore, the subsequent decrease in the number of cases following the recall of Diet $\mathrm{Y}$ was additional evidence of the strong association between the consumption of Diet $\mathrm{Y}$ and the development of AIME.

The data for 1,160,940 dogs between January 1, 2012, and February 15, 2018, were available from VetCompass Australia, of which 1,312 dogs aged > 6 months had the term megaesophagus associated with their medical records (in an attempt to exclude dogs with congenital ME). The data from these dogs were then analyzed to ensure the case definition was met. Prior to 2016, the incidence of ME was stable at a median of 0.08 cases $/ 100,000 \mathrm{dogs} / \mathrm{d}(3.8 \mathrm{dogs} / \mathrm{d}$ in a population of 4.8 million dogs). In 2017 , the marked increase in the number of dogs diagnosed with AIME by Australian veterinarians confirmed the outbreak (Figure I). The superimposed data from the case-control study showed a strong correlation between dogs that consumed Diet Y and a diagnosis of AIME. Data were not collected on the $>96 \%$ of dogs in the general population that were fed Diet Y but did not develop clinically apparent disease.

The assessment of breed, immunoreactivity, and environmental exposures would have been helpful in identifying possible cofactors contributing to or protecting dogs from the development of AIME. Mars Petcare Australia had been providing Diet $\mathrm{Y}$ to 20 independent breeders for the feeding of 80 dogs of various breeds but mostly large and medium breeds (eg, large-breed dogs such as Bullmastiff, Great Dane, and German Shepherd Dog; and medium-breed dogs such as Australian Cattle Dog, Basset Hound, Border Collie, and West Highland White Terrier). These breeders fed Diet $\mathrm{Y}$ from lots that had been associated with AIME in other dogs. Some breeders continued to feed Diet $\mathrm{Y}$ despite the recall and recommendation to cease feeding it, yet none of these dogs were reported to have had developed clinical signs of ME.

The UoM investigation also included nutritional and toxicological analyses of tissue samples from affected dogs and of Diet $\mathrm{Y}$ that had been returned from the owners of affected dogs and from personnel at the working dog facility. Uncommon ingredients, compared with other dog foods, in Diet Y included tuna meal (dry-matter basis, $4.4 \%$ ), maize gluten (30\%; approximately $2 \mathrm{X}$ the typical amount in dog foods), pilchard (sardine) autolysate (8.6\%), and a skin nutrient supplement of amino acids and $B$ vitamins. Nutritional assessment of Diet $\mathrm{Y}$ was undertaken at 2 reference laboratories, ${ }^{\mathrm{j}, \mathrm{k}}$ and measured analytes were found to be in accordance with the guidelines stated by the AAFCO and FEDIAF (a trade body that represents the European pet food industry and consists of members from 18 countries and 5 companies, including Mars Petcare).

Toxicological analyses were initially broad for any possible neurotoxins and then focused for possible neurotoxins associated with the ingredients in Diet Y. Toxicological analyses were performed at 2 certified external laboratories ${ }^{\mathrm{j}, \mathrm{k}}$ with various analytic methods, including liquid chromatography-mass spectrometry, gas chromatography-mass spectrometry, high performance liquid chromatography, and inductively coupled plasma mass spectrometry for standard food toxicants (including carbamate, organophosphate, and organochloride pesticides), mycotoxins, acrylamide, hexane, biogenic amines, sorbic acid, butylated hy-

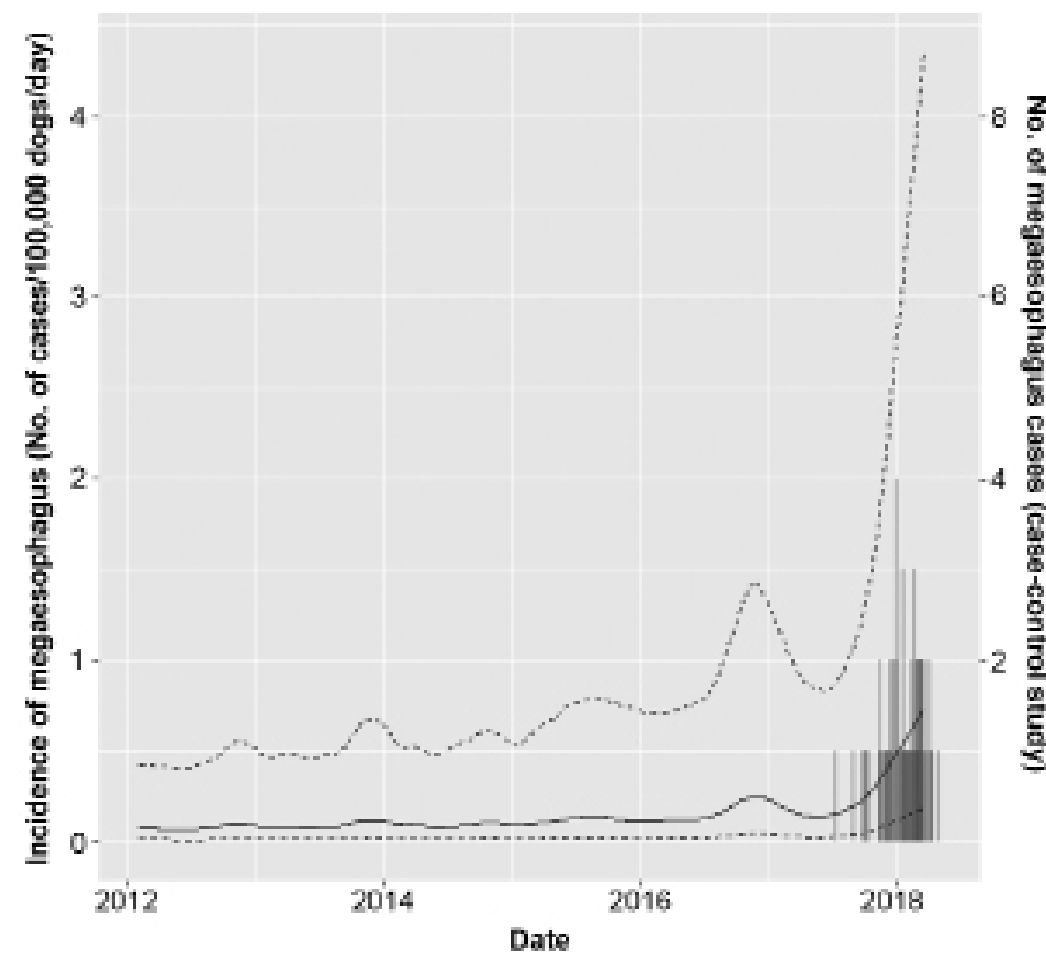

Figure I-Line plot showing the incidence of canine ME, expressed as the number of cases per 100,000 dogs per day, from January I, 2012, to February 15, 2018 (inclusive), in Australia. The solid line shows the point estimate of ME incidence as a function of calendar date. The upper and lower dashed lines indicate the $95 \% \mathrm{Cls}$ for the incidence estimates. Superimposed on this plot is a frequency histogram showing date of onset of clinical signs for dogs with ME (cases) used in the case-control study. (Adapted from Renwick M, Stevenson MA, Wiethoelter $A$, et al. A case-control study to identify risk factors for adult-onset idiopathic megaoesophagus in Australian dogs, 2017-2018. BMC Vet Res 2020;16:157. Reprinted with permission.) 
droxyanisole, butylated hydroxytoluene, BEA, domoic acid, paralytic shellfish poison, and essential and nonessential metals. None of the analytes for which toxic concentrations were known were at toxic concentrations in any of the food and tissue samples. However, some analytes for which toxic concentrations were not known were detected in Diet $Y$, and the concentrations of some analytes were higher in Diet $Y$ than in the simultaneously evaluated control diets (supermarket-brand adult dog and adult cat foods).

Of interest were emerging mycotoxins or mycotoxins that are not regulated by country-specific regulatory agencies, including BEA, because of their unknown potential for neurotoxicosis and the high maize content of Diet Y. The mycotoxin BEA is produced by many Fusarium spp, which are natural contaminants of cereals and cereal-based products. Because of BEA's biological properties, BEA can act as an ionophore and increase ion permeability in biological membranes. Consequently, BEA has a toxic effect on cells of several cell lines and can induce oxidative stress at the molecular level. The European Food Safety Authority has concluded that acute exposure to BEA poses no human health risks, but little toxicological data are available regarding chronic exposure. ${ }^{9}$ One recent study ${ }^{10}$ reveals that BEA is a common contaminant in dry pet foods in China (positive, 32/33 [97\%] samples; mean, $0.019 \mathrm{mg} / \mathrm{kg}$ of food $[0.01 \mathrm{mg} / \mathrm{lb}]$; range, 0.002 to 0.153 $\mathrm{mg} / \mathrm{kg}$ [0.01 to $0.07 \mathrm{mg} / \mathrm{lb}])$. Measured BEA concentrations determined at a US-based laboratory ${ }^{1}$ from the food samples submitted by UoM ( $\mathrm{n}=5$; median 0.62 $\mathrm{mg} / \mathrm{kg}[0.28 \mathrm{mg} / \mathrm{lb}]$; range, 0.56 to $0.69 \mathrm{mg} / \mathrm{kg}[0.25$ to $0.31 \mathrm{mg} / \mathrm{lb}])$ and MPA $(7 ; 0.107 \mathrm{mg} / \mathrm{kg}[0.05 \mathrm{mg} / \mathrm{lb}$; 0.05 to $0.13 \mathrm{mg} / \mathrm{kg}$ [0.02 to $0.06 \mathrm{mg} / \mathrm{lb}])$ initially differed, but that difference was not noted with reanalysis of a subset $(n=7)$ of samples (UoM: median, 0.097 $\mathrm{mg} / \mathrm{kg}[0.044 \mathrm{mg} / \mathrm{lb}]$, and range, 0.06 to $0.13 \mathrm{mg} / \mathrm{kg}$ [0.03 to $0.06 \mathrm{mg} / \mathrm{lb}]$; MPA: median, $0.11 \mathrm{mg} / \mathrm{kg}[0.05$ $\mathrm{mg} / \mathrm{lb}$ ], and range, 0.057 to $0.15 \mathrm{mg} / \mathrm{kg}$ [0.026 to 0.07 $\mathrm{mg} / \mathrm{lb}])$. The relevance of the variable BEA concentrations was unknown because its concentrations were within the range that was considered safe.

In parallel with the UoM investigation, MPA launched an extensive internal review of Diet $\mathrm{Y}$, including its production facilities, raw materials, packaging, supply chain, and inbound logistics (transport, storage, and delivery of goods). The production of Diet $Y$ followed a typical dry extrusion process in which dry ingredients, including essential vitamins and minerals, for each batch were weighed, mixed, and then pushed into a high-pressure cooker-extruder. Steam and other liquids were then injected into the extruder. The moist kibbles that exited the extruder were cooled, dried, and coated with palatants (ingredients that are often added to pet foods and treats to improve taste) and oils before being packaged. Diet Y was typically produced once per month and packed directly into its final packaging of 3-, 8-, and 15-kg (6.6-, 17.6-, and 33-lb) bags, with the predominant size determined by market demand (commonly 15-kg bags).
The shape and size of each kibble of Diet Y were not different from the other diets under the same brand name, with each kibble of triangle shape and medium size $(18 \mathrm{~mm})$, suitable for dogs of various sizes. Mean moisture level for Diet Y was low, so it was 50\% crispier than other MPA diets. Crispiness was analyzed through a validated mechanical texture technique that involved subjecting a piece of kibble to deformation in a controlled manner and then measuring the force response of the kibble under the applied deformation conditions. Texture analysis showed a lower fracture force for the kibble of Diet Y, indicative of a 17\% softer kibble than typical kibble of other diets. Diet $\mathrm{Y}$ was also slightly acidic ( $\mathrm{pH}, 4.5$ [pH of most pet foods, $\left.\left.{ }^{3} 5.0\right]\right)$, presumably because of the inclusion of fish-based ingredients.

Samples of Diet Y underwent nutrient analyses, and results were compared with nutrient standards set by the Australian Standard AS:5812 (2017), AAFCO, and FEDIAF, and findings showed that Diet $Y$ met their standards. Only 1 nutrient, vitamin D, from 1 analysis was found to be at a higher concentration than expected yet still within acceptable specifications. This result was assumed to be because of a variation in the type of tuna meal. Data analyses identified several parameters (fatty acid profile and biogenic amines, mycotoxin, and vitamin A concentrations) that had minor fluctuations in their concentrations during 2017 but remained within acceptable limits and therefore not considered concerning. Ionophores (BEA, calcimycine, cezomycin, carbonyl cyanide m-chlorophenyl hydrazine, enniatin, gramicidin A, ionomycin, lasalocid, monensin, nigericin, nonactin, salinomycin, tetronasin, valinomycin, and narasin $)^{1}$ and marine toxins ${ }^{\mathrm{m}}$ were not detected, or their concentrations were within acceptable limits.

The working dogs seen at UoM had been fed Diet Y for $>2$ years without incident. Therefore, on the basis of available production and distribution data, ME was attributed to Brand Y produced after July 2017 and fed from mid- to late-October 2017. Multiple food samples were tested from lots that had production dates of May 2017 to March 2018, prior to the date (March 24, 2018) when Diet $Y$ had been recalled and its production had ceased. A sample from each of 20 lots was analyzed for $>150$ substances, ${ }^{j, n-q}$ including amino acids, biogenic amines (added to preserve freshness), fatty acids, metals, minerals, mycotoxins, pesticides, and vitamins (Supplementary Table SI, available at: avmajournals.avma.org/doi/ suppl/10.2460/javma.259.2.172). The analyses indicated lot-to-lot variations that were major and minor yet still within acceptable limits in several analytes concurrent with a change in the supplier of maize gluten in mid-2017. The major proteins-tuna meal, pilchards, and maize gluten-were sourced from American Samoa, Australia, and the United States and Australia, respectively. The main starch, rice, was sourced from Australia and Thailand. Other minor but essential ingredients including vitamin and mineral blends were sourced from various countries, including Australia, Europe, the United States, and China.

All packaging materials for Diet $\mathrm{Y}$ were sourced from China and Germany. Ninety-two percent of dog owners in the UoM case-control study reported feed- 
ing their dogs from 15-kg bags of Diet Y, presumably because their dogs were predominantly large-breed dogs or dogs from multidog households. The $3-\mathrm{kg}$ bag packaging differed in its material structure and formulation specifications, compared with the packaging of the $8-\mathrm{kg}$ and $15-\mathrm{kg}$ bags. Samples of packaging for each bag size were analyzed for chemicals and possible migration of aromatic amines (from the packaging to the kibble). Diet $\mathrm{Y}$ packaging passed the chemical analysis and the test for migration. All packaging was in line with standards in Australia, the European Union, and the United States. Furthermore, the packaging was used across the full range of Diet $\mathrm{Y}$ foods, and no other diets under the same brand were found to be associated with ME.

All MPA records were reassessed and, with the assistance of suppliers, detailed analyses of the ingredients and production records were undertaken. In many instances, investigations were conducted by use of a material quality management program that included a review of the entire supply chain, beginning with the origin site (farm) of raw materials included in Diet $\mathrm{Y}$ to determine whether any important changes occurred in the farming of these raw materials; the weather conditions during farming, which may have introduced natural risks (eg, floods or bushfires can lead to mycotoxin production); the pesticide regimens; and the consistency in handling or additional processing of these raw materials.

The review of the local supply chain did not identify a likely chemical contaminant or plausible food safety or security risk. However, the tuna meal supply chain was identified as posing the greatest contamination risk because its supply chain was the most complex with multiple logistic steps. Contamination with other fish species, such as salmon, was unexpectedly confirmed. Despite this contamination, tuna meal was not implicated as a causative factor because all analyses for marine toxins were negative. ${ }^{n}$

Seasonal availability of ingredients, such as maize gluten meal, created the need to alternate supply between Australian and US sources. Mars Petcare Australia's extensive testing of the maize gluten from both the Australian and US sources was within specification, but analyses indicated that the 2 sources had slightly different compositions. Specifically, the moisture level was 1.5\% in the Australian-sourced maize gluten meal versus $8 \%$ to $12 \%$ in the US-sourced maize gluten meal. The maize gluten that was in Diet Y during the period of the ME outbreak was slightly higher in the concentration of fumonisin mycotoxins; however, all concentrations, including BEA, remained within acceptable limits.

Inspection of the manufacturing site for Diet $\mathrm{Y}$ in Bathurst, NSW, Australia, and review of the manufacturing process were undertaken. Manufacturing conditions and interruptions and deviations from the manufacturing process were scrutinized, and all conditions, interruptions, and deviations were found to be within specification. Additionally, MPA considered the possibility of intentional food tampering, but with the distribution pattern and duration of the ME outbreak and following a thorough review of factory and supply chain logistics, intentional food tampering was highly unlikely. Furthermore, handling and distribution of Diet $Y$ that was fed to affected dogs were not different from the handling and distribution of Diet Y that was fed to unaffected dogs.

\section{Discussion}

To the authors' knowledge, no association between dog food and the development of ME had been reported prior to these 2 outbreaks. The prevalence of AIME in dogs is unknown, yet anecdotally most veterinarians consider it to be low. Although the baseline data from VetCompass Australia indicate that approximately 3.7 cases of AIME/d were diagnosed among 4.8 million dogs prior to the outbreak of 20172018 in Australia, the incidence in other countries is unknown. The baseline data from VetCompass Australia may be an underestimation of the true prevalence of AIME, given that the data reflect only dogs whose owners sought veterinary care and diagnostic testing to determine the cause of their dogs' clinical signs (eg, regurgitation).

In both the Latvian and Australian outbreaks, epidemiological evidence was clear for a strong association between AIME and feeding of specific dog foods. Although a detailed comparison of dog food formulations was not possible (ingredients and their sources and recipes were unavailable for Brand $\mathrm{X}$ ), Brand $\mathrm{X}$ and Diet Y presumably had different ingredient sources (eg, European vs Australian) and combinations.

One striking difference between the 2 outbreaks was the presence of a clinical PNP in the Latvian dogs but not in the Australian dogs. In the Australian case dogs, 6 were examined by a veterinary neurologist, and no weakness or other signs of PNP were noted. One dog that underwent general anesthesia had laryngeal paralysis that may have been an early manifestation of PNP; however, this diagnosis was subjective, and the dog did not have clinical signs consistent with laryngeal paralysis or later develop a PNP. Peripheral nerves were examined histologically and with EMG and MNCV in 1 case and histologically in 3 other cases; findings were unremarkable except for distal axonopathy in the recurrent laryngeal nerve in $1 \mathrm{dog}$. One possible explanation for the differences in the observation of a PNP between the 2 outbreaks was that the dogs in Latvia were fed Brand $\mathrm{X}$ for a longer duration ( 6 to 12 months vs 1 to 3 months in duration for dogs fed Diet Y in Australia) prior to diagnosis of AIME; however, this was an unlikely reason because some Australian case dogs may have also been fed Diet $Y$ for a similar duration prior to AIME diagnosis and did not develop a PNP. Another possible explanation is that Brand $\mathrm{X}$ was deficient in vitamins B1, B6, and B12, whereas Diet $Y$ was not deficient in these $B$ vitamins. This deficiency may have predisposed to the development or worsened the clinical progression of PNP in the Latvian case dogs. 
Diet $\mathrm{Y}$ was the only diet implicated in the Australian incident. Diet Y was launched, produced, and distributed exclusively in Australia starting in 2012. Batches of Diet Y were produced approximately once per month and constituted a nutritionally complete and balanced diet to assist in the management of dogs with skin sensitivity. The proteins in Diet $\mathrm{Y}$ were primarily fish, maize gluten, and rice, whereas the proteins in most Australian dog foods were chicken, beef, lamb, and wheat (gluten). The Diet $\mathrm{Y}$ recipe had been consistent since the diet's launch in 2012; however, the source of some ingredients varied because of unavoidable seasonal conditions or supply chain problems. According to the records of phone calls received through MPA's customer care service, no severe adverse reactions associated with Diet $Y$ had been reported prior to 2017.

Because Diet Y was the only diet among diets under the same brand name that was associated with AIME, specific attention was focused on 3 unique raw materials-tuna meal, maize gluten, and pilchardsthat were at higher concentrations in Diet $Y$ than most other commercial dog foods in Australia. From tracing product codes, the batches fed to the case dogs were produced after July 2017. A causative agent was speculated to have been inadvertently included in Diet $\mathrm{Y}$ in mid-2017 concurrent with a change in a raw material. When combined with genetic or environmental factors, such as warmer temperatures and use of flea, tick, and heartworm preventives during September and October, the ME outbreak, affecting 3.7\% of the dog population that consumed Diet Y, may have been triggered. However, the epidemiological study did not identify an association with the use of flea, tick, or heartworm preventives. One change to the raw materials in mid-2017 was the source of the maize gluten, which constituted $30 \%$ of the diet formulation. Several minor analytic changes in the concentrations of tyramine, vitamin A, total fat, fumonisin, and zearalenone were noted concurrent with the change in the source of the maize gluten, but their concentrations remained within acceptable limits.

The high incidence of AIME of 30\% in dogs from the same household (presumably dogs were fed from the same bag of Diet Y), compared with the incidence of $3.7 \%$ of the general dog population that was fed Diet Y, was supportive of nonhomogeneous distribution of a causative agent within bags from the same batch or a cofactor in the environment. This may have posed a problem in testing the kibble from random bags or from a bag fed to a case dog because a causative agent may have been missed, especially if it was at a low concentration. Another possibility to explain the incidence differences included case dogs' common exposure to an environmental trigger, such as a chemical or virus, or case dogs having an individual immunologic, genetic, or microbiome variation that may have contributed to a reaction to an unknown component in Diet $Y$ and subsequent development of AIME.

In February 2019, MPA convened a multidisciplinary team of international scientific experts for a 2-day workshop to review the available data, discuss possible root causes, outline the research opportunities and imperatives, and establish a best-plan scenario for preventing, identifying, and managing future outbreaks. Before this workshop, the experts were provided access to all MPA's investigation data from the Australian outbreak, reports from the UoM investigation, and outcomes of the studies conducted in Latvia that implicated Brand $\mathrm{X}$ with the development of AIME. Speculative reasons for the association between Brand X or Diet Y and the development of AIME were collected and shared among the experts for creating a prioritized list of possible next steps.

The experts' input supported the extensive work done in 2018 and underscored the complexity of the situation, and the experts concluded that identifying a single cause was unlikely. Given that only $3.7 \%$ of dogs that were fed Diet Y had confirmed AIME in the Australian outbreak, the cause was unanimously considered most likely to be multifactorial with individualized sensitivity. Other factors may have included breed, immunosensitivity or allergic reaction, cumulative exposure to a yet unidentified toxic component of the diet, or abrasive kibble texture. Despite extensive investigations, several other food-associated illnesses in people and animals remain unresolved; these include the role of diet in Alzheimer's disease, ${ }^{11}$ Fanconi-like syndrome in dogs fed jerky treats, ${ }^{12}$ neurologic disease in cats fed irradiated dry cat food, ${ }^{13}$ and dilated cardiomyopathy in dogs fed grain-free dog foods. ${ }^{14,15}$ Therefore, a cause of diet-associated AIME may not be identified without greater awareness and early detection of disease as well as a thorough epidemiological investigation and sample collection of dietary raw ingredients and tissues from affected dogs.

On the basis of the investigations of these 2 outbreaks, the major informational gaps identified include the following:

- The role of the interaction of various factors in the induction of toxicosis or immunoreactivity. Although toxicological analysis did not reveal a cause and concentrations of possible toxins that were analyzed were within acceptable limits, predicting whether or how these toxins may interact when they are combined and whether dogs are chronically exposed would be exceeding difficult. Although dry pet foods are typically sterile because of the high temperatures used in extrusion, incidents of bacterial and fungal contamination of pet foods may contribute to various disease processes. Potential microbial toxins or molecular disruptors were considered possible but unlikely.

- Whether the crispy texture of dry kibble contributed to the disruption of the esopbageal epithelium. Four of 6 dogs in the Australian outbreak had mild to moderate microulceration of their esophageal mucosa that may have been secondary to delayed transit 
of food or to the kibble texture. In contrast, esophagitis was noted in only 4 of 30 dogs in the Latvian outbreak. The texture of Brand $\mathrm{X}$ kibble was untested but was thought to be normal and therefore implied a different mechanism for the development of esophagitis. In Australia, Diet Y kibble had been safely consumed for 5 years prior to the outbreak without owner reports that their dogs were affected by oral cavity abrasions or illness. The frequency of esophageal microulceration in dogs with ME is unknown; however, microulceration was speculated to have been a possible route of local absorption of a yet unidentified toxin.

- Whether factors such as environment, breed of dog, presence of allergies, and exposure to various chemicals predispose dogs to diet-associated AIME. Whether Australian case dogs had preexisting immunosensitivities that required hypoallergenictype diets is unknown. These dogs may have had an immunologic reaction to a component of Diet Y. Importantly, no information could be collected on unaffected dogs that had also consumed Diet Y. Whether these unaffected dogs (without apparent ME) were nonsusceptible breeds, lacked an immunosensitivity, or had an unknown protective effect remains unclear. That the cause was heterogeneously distributed in the food was also plausible.

- The lack of information on AIME in dogs. Dogs are inherently susceptible to developing ME, and models of spontaneous development of ME in animals do not exist. In addition, in vivo studies of dogs with acquired $\mathrm{ME}$ are rare ${ }^{16}$ expensive, and challenging to execute with ethical constraints in line with the appropriate care and use of animals. Acquired idiopathic ME likely has variable causes, but data from these 2 outbreaks were insufficient to determine whether neurologic lesions are similar among dog breeds and to determine the frequency with which AIME is associated with PNP. Of equal importance, comprehensive histologic assessments of the affected esophageal mucosa were lacking for the case dogs of these outbreaks, and this lack of assessments precluded an ability to determine whether esophagitis and esophageal erosions are common for dogs with AIME.

A better understanding of the possible causes of AIME is clearly needed to inform veterinarians such that they can identify and report an outbreak early. Ways to achieve a better understanding include studies that expand the epidemiological investigation, studies that better characterize AIME such that the optimal biological samples are harvested from affected dogs and the samples are optimally preserved, and studies that assess the impact of kibble texture on a dog's esophageal mucosa. To that end, the authors have engaged several veterinary stakeholders, including the Australian Veterinary Association, the Latvian Veterinary Medical Association, the World Small Animal Veterinary Association, and the Comparative Gastroenterology Society.

Further research considerations discussed among stakeholders include a genome-wide association study to determine whether single nucleotide polymorphisms are present in dogs of certain breeds that predispose them to ME, metagenomics sequencing for microbes and associated toxins, metanalyses of all data to identify possible case scenarios, and in vitro studies to determine the toxic effect of various substances on esophageal mucosal cell cultures. These studies are extensive and expensive, and limited blood and tissue samples remained available from affected dogs for further analyses.

In the Australian outbreak, a group of working dogs served as a sentinel for the possible association of AIME with consumption of a dog food. Without this sentinel group, this outbreak may have gone unrecognized for months to years, as it did in Latvia. Once pet dogs were also found to be affected with AIME in association with the same diet as that of the working dogs, recall of the diet was immediate, thus limiting additional dogs from being affected. A better surveillance system for early identification of pet illnesses is needed.

More pet food regulations have been suggested but without a clear understanding of the dietary triggers for pet illness, regulations alone may not prevent future diet-associated illness, including AIME. The US CDC has proposed that clear steps be outlined for the identification, management, and control of an outbreak. A scenario-planning protocol has been proposed for future outbreaks of AIME (Appendix).

\section{Acknowledgments}

Drs. Hurley, Lulham, and Naar are associates of Mars Inc, of which MPA is a subsidiary unit. The other authors declare that there were no conflicts of interest.

The authors acknowledge the work of the Latvian investigative team and veterinarians; international experts; the clinical and pathology staff at U-Vet Werribee Animal Hospital, UoM; and all owners and handlers whose dogs were enrolled in various studies discussed in this report.

\section{Footnotes}

a. Matīse-VanHoutan I, Lusis I, Viltrop A, et al. Food-associated megaesophagus/polyneuropathy outbreak in Latvian dogs: summary of clinical signs, epidemiology, and toxicological investigation (abstr), in Proceedings. 40th Annu Meet Symp Nordic Soc Vet Pathol 2017;31.

b. Brand $\mathrm{X}$ was a dry dog food produced and distributed in Latvia. The brand name was withheld for legal reasons.

c. Google, Alphabet Inc, Mountain View, Calif.

d. Veterinary Diagnostic Laboratory, Iowa State University, Ames, Iowa.

e. NP Analytical Laboratories, St Louis, Mo.

f. Hill's Science Diet Adult Sensitive Stomach \& Skin dog food, Hill's Pet Nutrition Pty Ltd, Sydney, NSW, Australia.

g. Laboklin, Bad Kissingen, Germany. 
h. Company data included in court papers but not publicly available.

i. Advance Dermocare, MPA, Southbank, VIC, Australia.

j. National Measurement Institute, Port Melbourne, VIC, Australia.

k. Agrifood Technology, Werribee, VIC, Australia.

1. Lawrence Livermore National Laboratory LLC, Livermore, Calif.

m. Cawthron Institute, The Wood, Nelson, New Zealand.

n. Romer Labs Singapore Pte Ltd, Singapore.

o. BVAQ Pty Ltd, North Melbourne, VIC, Australia.

p. Kemin Nurisurance Inc, Deakin University Waurn Ponds, VIC, Australia.

q. Eurofins, Dandenong South, VIC, Australia.

\section{References}

1. Matīse-VanHouten I. I did my research, blew the whistle and found myself at war. Available at: tedxriga.com/researchblew-whistle-found-war/. Accessed Mar 30, 2019.

2. Mansfield C, Renwick M, Stevenson M, et al. Summary of the University of Melbourne investigation into megaoesophagus and its association with pet food. Available at: www.u-vet.com.au/news/megaesophagus-and-pet-food/ megaoesophagus-and-pet-food-report14122018. Accessed Mar 30, 2019.

3. Renwick M, Stevenson MA, Wiethoelter A, et al. A case-control study to identify risk factors for adult-onset idiopathic megaoesophagus in Australian dogs, 2017-2018. BMC Vet Res 2020;16:157.

4. McGreevy P, Thomson P, Dhand NK, et al. VetCompass Aus tralia: big data and real-time surveillance for veterinary science. Animals (Basel) 2017;7:74.

5. Harris PA, Taylor R, Thielke R. etc. Research electronic data capture (REDCap): a metadata-driven methodology and workflow process for providing translational research informatics support. J Biomed Inform 2009;42:377-381.

6. Harris PA, Taylor R, Minor BL. etc. The REDCap consortium building an international community of software partners J Biomed Inform 2019;95:103208.

7. Oberbauer AM, Belanger JM, Bellumori T, et al. Ten inherited disorders in purebred dogs by functional breed groupings. Canine Genet Epidemiol 2015;2:9.

8. Gaynor AR, Shofer FS, Washabau RJ. Risk factors for acquired megaesophagus in dogs. $J$ Am Vet Med Assoc 1997;211:1406-1412.

9. Mallebrera B, Prosperini A, Font G. In vitro mechanisms of beauvericin toxicity: a review. Food Chem Toxicol 2018;111:537-545.

10. Shao M, Li L, Gu Z, et al. Mycotoxins in commercial dry pet food in China. Food Addit Contam Part B Surveill 2018; $11: 237-245$

11. Klotz K, Weistenhöfer W, Neff F. The health effects of aluminum exposure. Dtsch Arztebl Int 2017;114:653-659.

12. Thompson MF, Fleeman LM, Kessell AE. Acquired proximal renal tubulopathy in dogs exposed to a common dried chicken treat: retrospective study of 108 cases (2007-2009). Aust Vet J 2013;91:368-373.

13. van den Ingh TSGAM, Grinwis GCM, Corbee RJ. Leukoencephalomyelopathy in cats linked to abnormal fatty acid composition of the white matter of the spinal cord and of irradiated dry cat food. J Anim Pbysiol Anim Nutr (Berl) 2019;103:1556-1563

14. Mansilla WD, Marinangeli CPF, Ekenstedt KJ. Special topic: the association between pulse ingredients and canine dilated cardiomyopathy: addressing the knowledge gaps before establishing causation. J Anim Sci 2019;97:983-997.

15. McCauley SR, Clark SD, Quest BW, et al. Review of canine dilated cardiomyopathy in the wake of diet-associated concerns. J Anim Sci 2020;98:skaa155.

16. Satchell PM, McLeod JG. Megaoesophagus due to acrylamide neuropathy. J Neurol Neurosurg Psychiatry 1981;44:906-913.

17. University of California-San Diego Comparative Neuromuscular Laboratory. Sample requirements. Available at: vetneuromuscular ucsd.edu/samplerequirements.html. Accessed Jan 7, 2018. 


\section{Appendix}

Ten steps of a scenario-planning protocol proposed for identification and investigation of future outbreaks of pet food-associated AIME.

\section{Step}

Identify investigation team and resources

Establish existence of an outbreak

Verify the diagnosis

Construct case definition

Find cases systematically and develop line listing

Perform descriptive epidemiologic investigation and develop hypotheses

Evaluate hypotheses and perform additional studies as necessary

Implement control measures

Communicate findings

Maintain surveillance

\section{Description}

In the event of an issue involving animals and potential food-associated illness, required experts may include those in veterinary medicine, nutrition, toxicology, neurology, pathology, and epidemiology. Resources to conduct thorough clinical and product investigations are critical.

An outbreak is an increase in the incidence of disease with relation to (known) baseline incidence, in association with signs of common disease patterns, infectious or otherwise, medications, food brands and types (eg, grain free), and geographic region. If a sentinel group of animals is experiencing an increase of disease within a population, an epidemiological exploration of common factors, exposures, dietary history, and geographies is crucial to elucidate contributing factors.

Clinical cases must be verified to ensure they are appropriately included in the case numbers. Thoracic radiography or videofluoroscopy is necessary to confirm esophageal dilation and decreased contractility, changes consistent with ME. Also, further diagnostic testing should be performed to rule out known causes of ME, such as hypothyroidism, hypoadrenocorticism, myasthenia gravis, toxicoses (eg, acrylamide, heavy metals, and organophosphates), polyneuropathies, and polymyopathies.

The case definition has 3 main components-individual (age, breed, body weight, neuter status, and sex), place (housing and living space and exposures), and time (time frame concurrent with outbreak).

Cases may be identified through word of mouth by dog owners and veterinarians, complaints filed with a manufacturer by dog owners and veterinarians, systems such as VetCompass Australia, PetFAST, and the FDA Veterinary Laboratory Investigation and Response Network. Data collection is essential to build the evidence and track spread and control of an outbreak. Additional crucial data include onset and duration of clinical signs, description of clinical signs, environmental or medicinal exposures, and dietary history.

Develop an epidemiological curve on the basis of the collected information and consider possible causes as follows: physical or mechanical (texture and format of product and effect on esophageal mucosa, ideally with controls looking at unaffected dogs consuming product in question), metabolic (rule out known causes of disease through testing and consider metabolomics, lipidomics, and proteomics), toxic (targeted testing [look for all known toxins that may fit the disease pattern] and nontargeted testing [look for product differences in various toxins known to cause disease and look at control or normal product to identify adulteration, contamination, altered substances, and denatured protein]), immunoreactive (evidence or history of allergy, immunologic disease, or immune stimulation in affected dogs vs control dogs fed product under investigation), genetic (ie, a genetic predisposition; examine differences in breed [size], sex, etc, between affected and unaffected dogs that consumed the product under investigation), infectious (exposure to viruses, bacteria, protozoal organisms, or prions, and note any environmental patterns of disease occurring simultaneously), and multifactorial (a combination of the aforementioned possible causes, determined through statistical correlations and metaanalyses). Once a mechanism has been identified, suspect substances can be evaluated with in vitro or in vivo studies depending on the identification of a disease model and consideration of ethics to proceed with the studies.

Conduct a case-control or cohort study. Consider logical workstreams to rule in or out the original hypotheses and conduct further analyses as warranted. Blood and tissue samples from the animal and products from affected batches as well as product returned from pet owners should be banked in appropriate containers and stored at appropriate temperatures if initial analyses do not reveal an exact or logical pattern and cause of disease. Collect whole blood and serum, feces for microbiome analysis, urine for metabolomic analysis, and tissues (obtained at necropsy) for histologic examination.*

If a common factor is identified, then immediate withdrawal of the offending material, including all common foods, medications, and environmental exposures, is paramount, regardless of whether the root cause has been identified. If a cause is not identified, then product should be withdrawn, and formulation, facilities, supply chain, ingredients, transport, distribution, and potential for sabotage should be evaluated.

Awareness of disease occurrence is critical to ensuring dog owners and veterinarians are aware of the possible outbreak, as well as for the critical collection of cases and case materials for further study. Communication may be through traditional media, social media, and veterinary or pet organizations to reach a broad audience.

The purpose of ongoing surveillance is to confirm that the outbreak is over and to determine the effectiveness of control measures. This becomes critical if the causative agent cannot be confirmed. Where connected databases exist (eg, VetCompass Australia and FDA Veterinary Laboratory Investigation and Response Network), retrospective analysis is possible, but ideally, a prospective monitoring process by use of machine learning (artificial intelligence) could be developed and implemented to identify any future recurrence.

*Full-thickness biopsies from the proximal, middle, and distal portions of the esophagus (including the lower esophageal sphincter); biopsies of the brain (cerebral cortex and brainstem), spinal cord, peripheral nerves, thyroid gland, larynx, diaphragm, and muscles of the peripheral portions of the limbs.

(Adapted from CDC. Principles of epidemiology in public health practice. Lesson 6: investigating an outbreak. Section 2: steps of an outbreak investigation. Available at: www.cdc.gov/csels/dsepd/ss 1978/lesson6/section2.html. Accessed Jan 7, 2018.) This protocol may also be applied to identification and investigation of other disease outbreaks. Muscle and nerve sample requirements are available online. ${ }^{17}$ 